\title{
Room-temperature continuous-wave electrically pumped InGaN/GaN quantum well blue laser diode directly grown on $\mathrm{Si}$
}

\author{
Yi Sun (1) ${ }^{1,2,3}$, Kun Zhou', Meixin Feng ${ }^{1,2}$, Zengcheng Li', Yu Zhou ${ }^{1,2}$, Qian Sun ${ }^{1,2}$, Jianping Liu ${ }^{1,2}$, Liqun Zhang ${ }^{1}$, \\ Deyao Li ${ }^{1}$, Xiaojuan Sun ${ }^{4}$, Dabing Li $\mathbb{C}^{4}$, Shuming Zhang ${ }^{1,2}$, Masao $\mathrm{Ikeda}^{1}$ and Hui Yang ${ }^{1,2}$
}

\begin{abstract}
Current laser-based display and lighting applications are invariably using blue laser diodes (LDs) grown on freestanding GaN substrates, which are costly and smaller in size compared with other substrate materials. ${ }^{1-3}$ Utilizing less expensive and large-diameter Si substrates for hetero-epitaxial growth of indium gallium nitride/gallium nitride (InGaN/GaN) multiple quantum well (MQW) structure can substantially reduce the cost of blue LDs and boost their applications. To obtain a high crystalline quality crack-free GaN thin film on Si for the subsequent growth of a blue laser structure, a hand-shaking structure was formed by inserting Al-composition step down-graded AIN/Al $\mathrm{Ga}_{1_{-x} \mathrm{~N}}$ buffer layers between GaN and Si substrate. Thermal degradation in InGaN/GaN blue MQWs was successfully suppressed with indium-rich clusters eliminated by introducing hydrogen during the growth of GaN quantum barriers $(\mathrm{QBs})$ and lowering the growth temperature for the p-type AlGaN/GaN superlattice optical cladding layer. A continuous-wave (CW) electrically pumped InGaN/GaN quantum well (QW) blue $(450 \mathrm{~nm})$ LD grown on Si was successfully demonstrated at room temperature (RT) with a threshold current density of $7.8 \mathrm{kA} / \mathrm{cm}^{2}$.
\end{abstract}

An InGaN/GaN QW blue LD as a key device component holds great potential for a wide range of applications ${ }^{4}$, including laser displays, laser-based automotive lighting, visible light communication ${ }^{5}$, undersea wireless communication $^{6}$, and high-density data storage ${ }^{7}$. Today, most InGaN/GaN QW blue LDs are homo-epitaxially grown on expensive 2-inch free-standing $\mathrm{GaN}$ substrates ${ }^{3}$, which leads to LDs costing orders of magnitude higher than lightemitting diodes (LEDs) grown on hetero-epitaxial substrates (such as sapphire, $\mathrm{SiC}$, or silicon). In addition, freestanding $\mathrm{GaN}$ substrates often suffer from non-uniformity in the offcut angle, defect density, and/or residual stress,

Correspondence: Qian Sun (qsun2011@sinano.ac.cn) or

Jianping Liu (jpliu2010@sinano.ac.cn)

${ }^{1}$ Key Laboratory of Nanodevices and Applications, Suzhou Institute of Nano-

Tech and Nano-Bionics (SINANO), Chinese Academy of Sciences (CAS), Suzhou 215123, China

${ }^{2}$ School of Nano Technology and Nano Bionics, University of Science and Technology of China, Hefei 230026, China

Full list of author information is available at the end of the article. affecting process reproducibility and production yield ${ }^{8}$. By contrast, Si substrates show several advantages in terms of wafer size, material cost, and uniformity, as well as the welldeveloped automotive processing line. By replacing costly and small-size $\mathrm{GaN}$ free-standing substrates with costeffective and large-diameter $\mathrm{Si}$ substrates, the cost of InGaN/GaN QW blue LDs can be reduced to the same level as LEDs, which will lead to further applications of LDs. Here, we demonstrate a continuous-wave $(\mathrm{CW})$ electrically pumped InGaN/GaN QW blue LD grown on a $\mathrm{Si}$ substrate operating at room temperature (RT).

$\mathrm{GaN}$ growth on Si typically involves a large mismatch in lattice constant and a huge misfit in coefficient of thermal expansion (CTE), which typically lead to a very high threading dislocation density (TDD) $\left(10^{9}-10^{10} \mathrm{~cm}^{-2}\right)$ and a significant tensile stress or, as is often the case, the formation of a micro-crack network, respectively ${ }^{9}$. Furthermore, edgeemitting LDs require low-refractive-index optical cladding layers for optical field confinement; however, the growth of

\section{(c) The Author(s) 2018}

(c) (i) Open Access This article is licensed under a Creative Commons Attribution 4.0 International License, which permits use, sharing, adaptation, distribution and reproduction cc) in any medium or format, as long as you give appropriate credit to the original author(s) and the source, provide a link to the Creative Commons license, and indicate if changes were made. The images or other third party material in this article are included in the article's Creative Commons license, unless indicated otherwise in a credit line to the material. If material is not included in the article's Creative Commons license and your intended use is not permitted by statutory regulation or exceeds the permitted use, you will need to obtain permission directly from the copyright holder. To view a copy of this license, visit http://creativecommons.org/licenses/by/4.0/. 


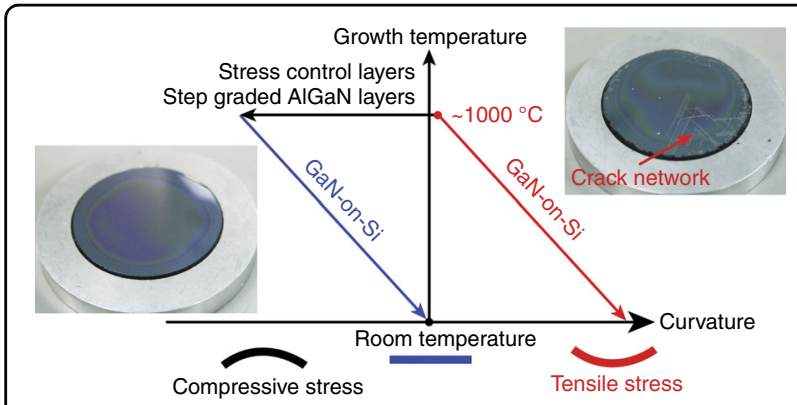

Fig. 1 Schematic diagram of the stress control for the hetero-epitaxy growth of crack-free GaN thin film on a Si substrate with Alcomposition step down-graded AIN/AIGaN buffer layers

AlGaN-containing optical cladding layers on $\mathrm{GaN}$ induces additional tensile stress. Therefore, defect control and stress engineering are critical for the realization of electrically pumped InGaN/GaN LDs grown on a Si substrate ${ }^{10}$.

These issues can be addressed by inserting a stack of Al-composition step down-graded $\mathrm{AlN} / \mathrm{Al}_{\mathrm{x}} \mathrm{Ga}_{1-\mathrm{x}} \mathrm{N}$ multi-layers between $\mathrm{Si}$ and $\mathrm{GaN}^{10-12}$, as shown in Fig. 1 . The compressive strain built up by the positive lattice mismatch in the GaN epitaxial film on the Si substrate was utilized to compensate for the tensile stress caused by the CTE mismatch during the cool-down period. Moreover, it was also found that threading dislocations (TDs) are more likely to incline and annihilate each other, especially at interfaces facilitated by the aforementioned compressive strain, resulting in a high crystalline quality GaN film that is epitaxially grown on a $\mathrm{Si}$ substrate ${ }^{12-14}$. The crystalline quality of the GaN film grown on $\mathrm{Si}$ was evaluated by double crystal X-ray rocking curve measurements (Fig. 2). The linewidths for both of the GaN (0002) and (1012) X-ray diffraction peaks were approximately 260 arcsec, corresponding to a low TDD $(\sim 5 \times$ $10^{8} \mathrm{~cm}^{-2}$ ). The limited difference between these two linewidths indicated a low density of edge type $\operatorname{TDs}^{15,16}$, which often serve as non-radiative recombination centers and, hence are more deterious to the internal quantum efficiency compared to screw and mixed type TDs ${ }^{17}$.

We have previously successfully demonstrated InGaN/ GaN QW violet LDs directly grown on Si substrate, lasing at a wavelength of $413 \mathrm{~nm}^{10}$. To further expand applications for laser displays and laser lighting, blue LDs with emission lasing wavelength longer than $450 \mathrm{~nm}$ are highly desirable. However, growing an InGaN/GaN QW blue LD structure on a Si substrate is more challenging: the longer the emission wavelength, the smaller the difference in refractive index between III-nitride materials ${ }^{18}$. Therefore, both the In composition in the InGaN waveguide layers and the $\mathrm{Al}$ composition in the AlGaN optical cladding layers must be increased to obtain a sufficient optical confinement for the realization of blue lasing ${ }^{19}$.

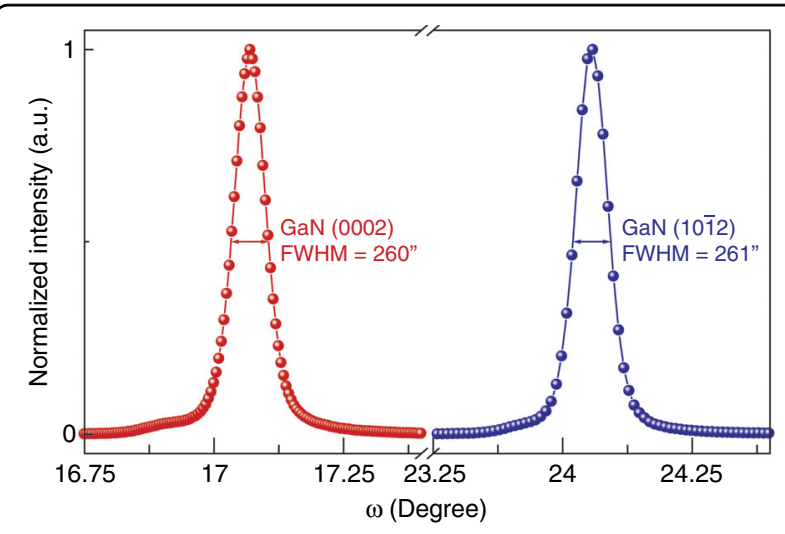

Fig. 2 Double crystal X-ray rocking curves for the GaN (0002) and (1012) diffraction peaks for a GaN film grown on a Si substrate. The value of full width at half maximum (FWHM) is labeled

However, thick InGaN waveguide layers, which are beneficial for optical confinement, often lead to strain relaxation. Hence, InGaN and $\mathrm{GaN}$ compound waveguide layers were used for both optical confinement and strain control (Supplementary Fig. S1). Moreover, the inserted $\mathrm{GaN}$ waveguide layers also served as a transition layer between the InGaN waveguide and the AlGaN optical cladding layer to mitigate the large lattice mismatch and improve the material quality (Fig. 3b). Simulation of the optical field distribution in the GaN-on-Si blue laser structure is shown in Supplementary Fig. S1. It is noted that most of the stimulated emission photons were confined in the active region, avoiding strong absorption by the Si substrate, which was evidenced by the near-field patterns (Supplementary Fig. S2). According to scanning transmission electron microscopy (STEM) cross-sectional images (Fig. 3), sharp interfaces for the individual layers indicated a high quality for the as-grown blue LD structure on Si.

On the other hand, a longer lasing wavelength also means a higher In composition in the MQWs, and threshold current density often increases dramatically with the In composition in MQWs ${ }^{20}$, especially when the dislocation density is relatively high $\left(\sim 5 \times 10^{8} \mathrm{~cm}^{-2}\right)$. Furthermore, the InGaN/GaN MQWs in blue LDs often suffer from thermal degradation due to the extended thermal process during the deposition of the upper AlGaN optical cladding layer at high temperature. Thermal degradation in InGaN/GaN MQWs in blue LDs was often observed through the formation of dark spots under micro-photoluminescence (micro-PL) imaging (Fig. 4a). The dark spots were related to thermally degraded regions in the MQWs, where metallic indium precipitates and voids are found ${ }^{21}$. It is generally believed that the precipitation of metallic indium and void formation originate from the decomposition of a fraction of indium-rich 

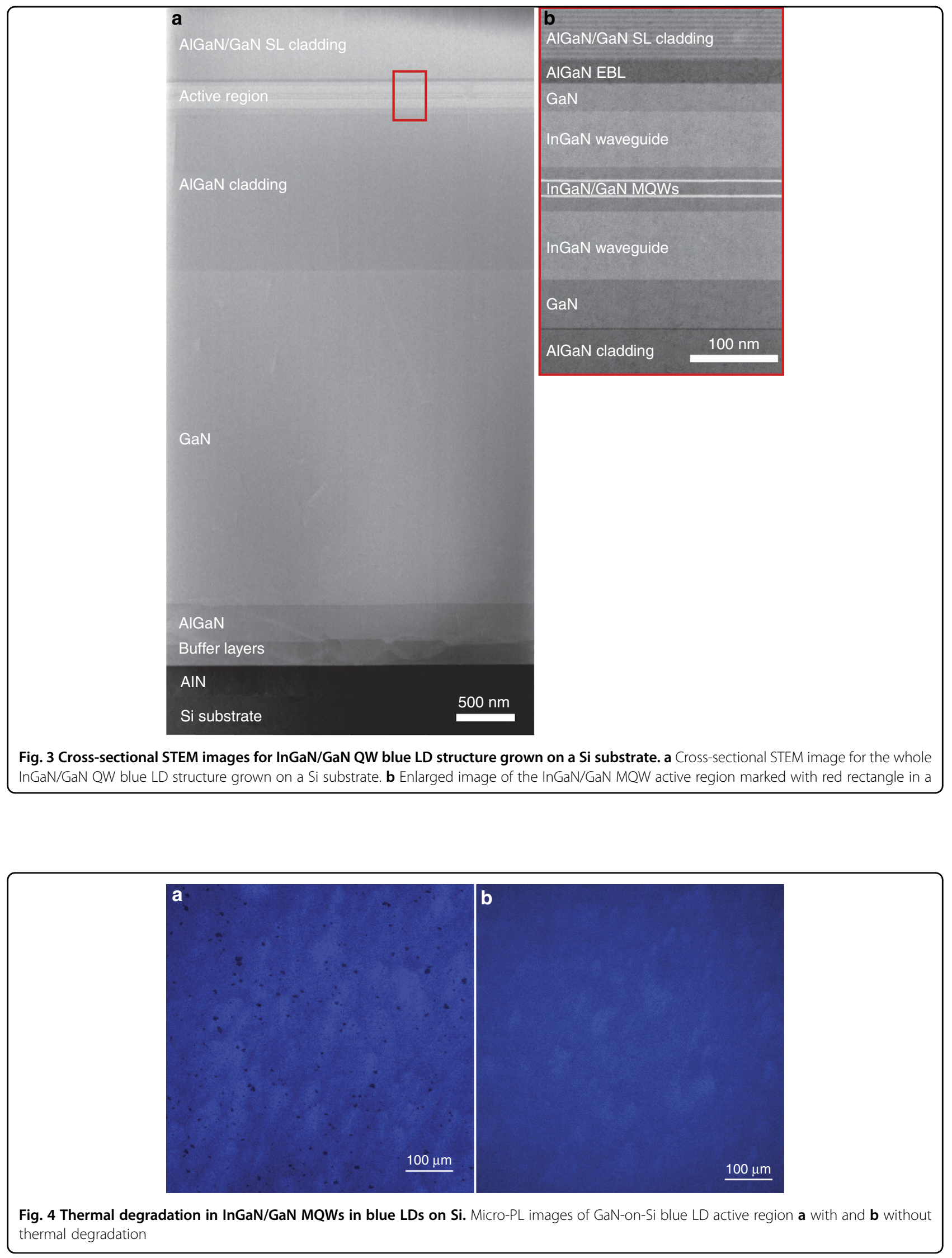
clusters in the $\mathrm{QW} /$ quantum barrier $(\mathrm{QB})$ interfaces or the QWs during the growth of the p-type optical cladding layer at high temperature ${ }^{22,23}$. Indium-rich clusters can form due to indium segregation ${ }^{24}$, indium accumulation around the $\mathrm{TDs}^{25}$, or indium-rich trench defects, as reported recently ${ }^{26,27}$. It is crucial to eliminate such defects and ensure that the InGaN/GaN blue MQWs are sufficiently robust to survive the extended thermal process during the upper AlGaN cladding layer growth.

To avoid thermal degradation in InGaN/GaN blue MQWs, we used $\mathrm{H}_{2}$ to eliminate In-rich clusters and also reduced the growth temperature for the upper AlGaN cladding layer ${ }^{28}$. Thermal degradation can be mitigated by reducing the thermal budget, which was realized by lowering the growth temperature of the thick p-AlGaN cladding layer from 978 to $970{ }^{\circ} \mathrm{C}$. It should be noted that a further reduction in growth temperature for the p-AlGaN material can cause a significant increase in series resistance and device operating voltage because of the unintentional incorporation of carbon impurities in the p-type materials ${ }^{29}$. More importantly, In-rich clusters must be removed to make the InGaN/GaN blue MQWs more robust. Since $\mathrm{H}_{2}$ can effectively etch away indium material from the growth surface, we intentionally introduced $600 \mathrm{sccm}$ of $\mathrm{H}_{2}$ into the carrier gas (corresponding to only $3 \%$ of the total gas flow rate) during the GaN QBs growth after the QW cap layer was deposited. The $\mathrm{H}_{2}$ source was switched off prior to the growth of the InGaN QW, ensuring no negative effect on indium incorporation during the InGaN QW growth. Meanwhile, a dualtemperature growth scheme was adopted when growing InGaN/GaN blue MQWs. The growth temperature of $\mathrm{GaN}$ QBs was $80^{\circ} \mathrm{C}$ higher than that of InGaN QWs, which can also assist in the elimination of indium segregation, indium accumulation, and indium-rich trench defects. As a result, thermal degradation of the InGaN/ GaN blue MQWs in the LD structure was effectively suppressed, as evidenced by the complete elimination of dark spots in the micro-PL imaging of the blue LD structure grown on $\mathrm{Si}$ (Fig. 4b).

The as-grown InGaN/GaN blue LD epitaxial wafer was fabricated into edge-emitting LDs (Fig. 5a, b) with a ridge width of $4 \mu \mathrm{m}$ and a cavity length of $400 \mu \mathrm{m}$ (see Materials and methods). The characteristics of the as-fabricated InGaN/GaN blue LD grown on Si under a CW electrical injection at RT are shown in Fig. 5c-f. The lasing
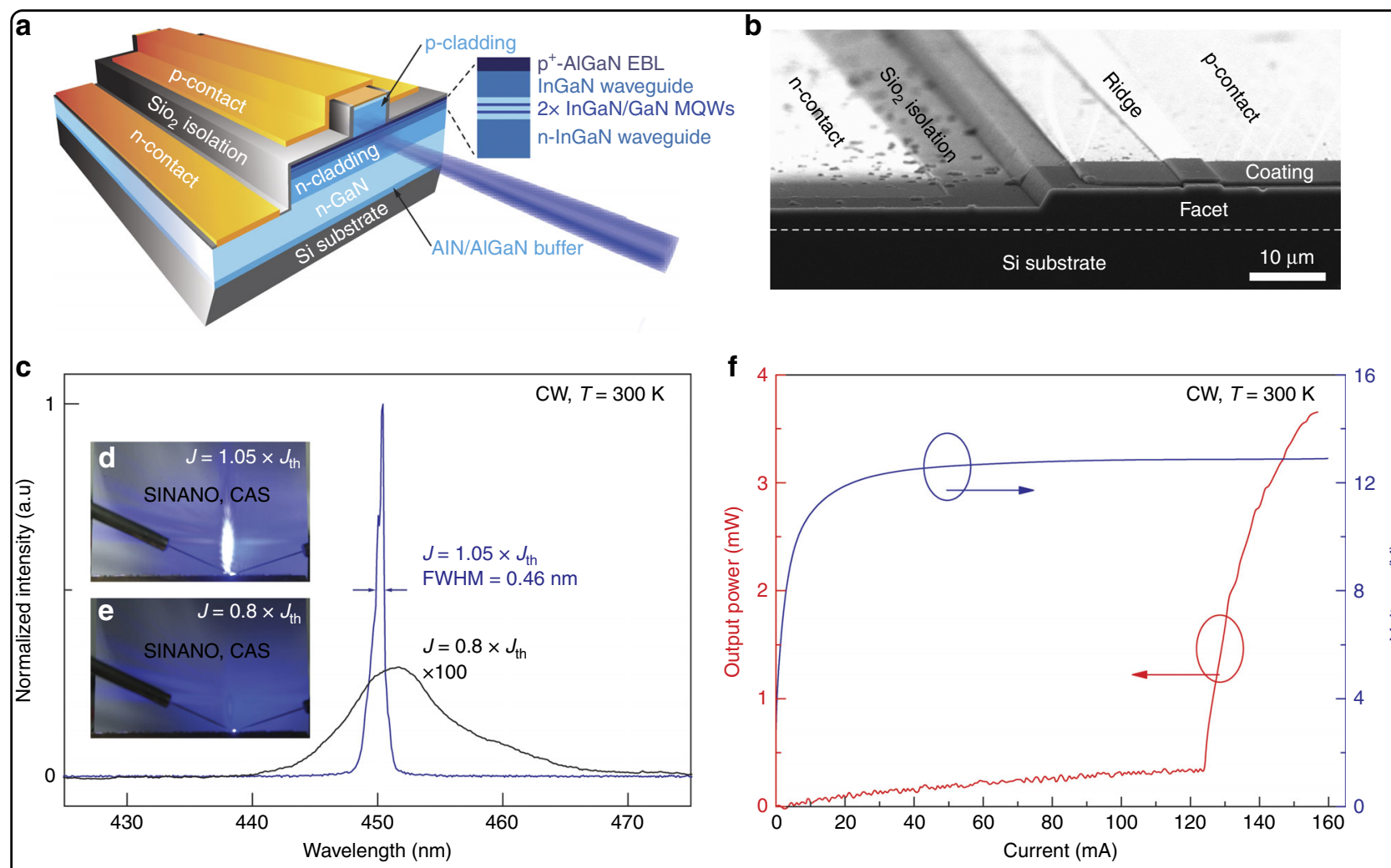

Fig. 5 Schematic architecture, scanning electron microscope (SEM) image and characteristics of blue GaN-on-Si LD. a Schematic structure of a blue LD directly grown onto a Si substrate. $\mathbf{b}$ Bird-view SEM image of a blue LD grown on Si. c EL spectra and FFPs of a blue LD grown on Si under a CW electrical pumping at RT. FFPs were observed $\mathbf{d}$ above and $\mathbf{e}$ below the threshold current by placing a sheet of paper in front of the LD front facet. $\mathbf{f} \mathrm{L}-\mathrm{I}-\mathrm{V}$ characteristics of the InGaN/GaN blue LD measured under a CW current injection at RT 
wavelength was $450.3 \mathrm{~nm}$ with a threshold current of $125 \mathrm{~mA}$, corresponding to a threshold current density of $7.8 \mathrm{kA} / \mathrm{cm}^{2}$. Fig. $5 \mathrm{c}$ shows the electroluminescence (EL) spectra of the LD under a $\mathrm{CW}$ electrical pumping below $(100 \mathrm{~mA})$ and above $(130 \mathrm{~mA})$ the threshold. When the injection current rose up from 100 to $130 \mathrm{~mA}$, the peak wavelength of the EL spectra was blue-shifted from 451.5 to $450.3 \mathrm{~nm}$, because of the screening of the quantumconfined Stark effect. At the same time, the full width at half maximum (FWHM) of the EL spectra quickly decreased to $0.46 \mathrm{~nm}$. The far-field patterns (FFPs) of the edge emission with a $\mathrm{CW}$ injection above and below the threshold current are shown in Fig. 5d, e, respectively. As a result of the asymmetric optical confinement in the longitudinal and latitudinal directions, an elliptical FFP (Fig. 5d) was observed elongated along the growth direction. The light-current-voltage (L-I-V) characteristics for the blue LD grown on Si under a CW electrical injection at RT are shown in Fig. 5f. The light output power increased much more rapidly when the injection current went beyond the threshold current of $125 \mathrm{~mA}$. The relatively high operating voltage for the as-fabricated LD was mainly due to the relatively large resistance of the p-type GaN/AlGaN superlattice optical cladding layer grown at a relatively low temperature with an unintentional doping of carbon impurities ${ }^{29}$, which, as compensation centers, greatly reduced the hole concentration. About $68 \%$ of the fabricated LDs could lase and the statistical results about the threshold current of the asproduced blue LDs grown on $\mathrm{Si}$ are shown in Supplementary Fig. S3, which presents a decent yield for the device processing.

It should be noted that the light output power under $\mathrm{CW}$ current injection at RT was measured following simple packaging with indium soldering. The slope efficiency of these devices shows a clear decrease after increasing the current injection to $130 \mathrm{~mA}$ (Fig. 5f). This reduced slope efficiency of the blue LD grown on Si under $\mathrm{CW}$ electrical injection was caused by a self-heating problem because of the relatively high threshold current density and the relatively high operation voltage of the asfabricated devices (Fig. 5f). The severe self-heating problem not only affected the LD performance but also shortened its lifetime (less than $1 \mathrm{~min}$ ), especially under $\mathrm{CW}$ operation. The self-heating problem can be mitigated by improving heat dissipation with a better package and by decreasing the threshold current and the operation voltage via optimization of the epitaxial structure and the growth conditions, as well as the LD fabrication process. In particular, the operating voltage can be greatly reduced by lowering the resistance of the p-type GaN/AlGaN superlattice optical cladding layer, which can be grown at a relatively lower temperature, but at a slower growth rate to suppress unintentional doping of carbon ${ }^{29}$. It is believed that the lifetime of the as-fabricated blue LDs grown on $\mathrm{Si}$ was also limited by the high $\mathrm{TDD}^{30}$. According to the previous reports, the lifetime of InGaNbased LDs can be improved from a few seconds ${ }^{31}$ to $300 \mathrm{~h}^{32}$, when the threshold current density was reduced from 9 to $4.2 \mathrm{kA} / \mathrm{cm}^{2}$, and the operation voltage was lowered from 8 to $4 \mathrm{~V}$. In addition, it has been reported that the lifetime of InGaN-based LDs can be further extended to more than $10,000 \mathrm{~h}$ when the TDD in the LD structure is reduced from $10^{8}$ down to $10^{6} \mathrm{~cm}^{-2}$ via an epitaxial lateral overgrowth of $\mathrm{GaN}^{30}$. For blue LDs grown on $\mathrm{Si}$, lowering of the operation voltage to approximately $4 \mathrm{~V}$ and reduction of the density of TDs from $10^{8}$ to $10^{6}$ $\mathrm{cm}^{-2}$ in $\mathrm{GaN}$ grown on $\mathrm{Si}$ through epitaxial lateral overgrowth is expected to decrease the threshold current density to less than $4 \mathrm{kA} / \mathrm{cm}^{2}$, with the lifetime possibly reaching a few hundred or even thousands of hours.

In conclusion, we have demonstrated a CW operation of InGaN/GaN QW blue LDs grown on a Si substrate at RT. The threshold current density was $7.8 \mathrm{kA} / \mathrm{cm}^{2}$ and the peak wavelength for the blue LD grown on $\mathrm{Si}$ was 450.3 $\mathrm{nm}$. These results highlight a promising technical approach for manufacturing InGaN/GaN blue LDs at substantially lower cost by using large-size $\mathrm{Si}$ as the epitaxial substrate. With further improvement in the lifetime of devices by reducing the threshold current and the operating voltage, together with reduction in the TD density by using epitaxial lateral overgrowth, GaN-on-Si technology holds great potential for the commercialization of InGaN-based blue LDs fabricated using costeffective, large-diameter Si substrates, further boosting wide applicability.

A commercial available metal-organic chemical vapor phase deposition system was employed for the growth of InGaN/GaN QW blue LD structure on a $\mathrm{Si}(111)$ substrate. Nitrogen and hydrogen served as carrier gases. Monosilane and bisethylcyclopentadienylmagnesium were adopted as the $\mathrm{n}$ - and p-type dopants, respectively. Trimethylaluminum, trimethylindium, trimethylgallium, and ammonia were utilized as precursors for aluminum, indium, gallium, and nitrogen, respectively. A 2-inch Si (111) substrate was firstly thermally cleaned before the growth of the hand-shaking buffer, which consisted of a 280-nm-thick AlN nucleation layer, a 180-nm-thick $\mathrm{Al}_{0.35} \mathrm{Ga}_{0.65} \mathrm{~N}$ layer, and a 310-nm-thick $\mathrm{Al}_{0.17} \mathrm{Ga}_{0.83} \mathrm{~N}$ layer. A 3- $\mu \mathrm{m}$-thick $\mathrm{n}-\mathrm{GaN}$ layer was then deposited on top of the hand-shaking buffer. Afterwards, an InGaN/ GaN QW blue LD structure was then grown, which consisted of a 1.4- $\mu$ m-thick n-type $\mathrm{Al}_{0.06} \mathrm{Ga}_{0.94} \mathrm{~N}$ optical cladding layer, a 55-nm-thick n-type $\mathrm{GaN}$ transition layer, a 75-nm-thick n-type $\operatorname{In}_{0.04} \mathrm{Ga}_{0.96} \mathrm{~N}$ waveguide layer, two pairs of 2.5-nm-thick undoped $\operatorname{In}_{0.15} \mathrm{Ga}_{0.85} \mathrm{~N}$ QW and 15-nm-thick undoped GaN QB layers, a 60-nm-thick undoped $\operatorname{In}_{0.035} \mathrm{Ga}_{0.965} \mathrm{~N}$ waveguide layer, a 30-nm-thick 
undoped GaN transition layer, a 20-nm-thick p-type $\mathrm{Al}_{0.2} \mathrm{Ga}_{0.8} \mathrm{~N}$ electron blocking layer, one hundred pairs of 3-nm-thick p-type $\mathrm{Al}_{0.14} \mathrm{Ga}_{0.86} \mathrm{~N}$ and 3-nm-thick p-type GaN superlattice optical cladding layers, and a 30-nmthick p-type $\mathrm{GaN}$ contact layer.

The as-grown blue LD epitaxial wafer was fabricated into edge-emitting LDs with a ridge width of $4 \mu \mathrm{m}^{10}$. The cavity length was $400 \mu \mathrm{m}$, which was formed by facet cleavage. Three and seven pairs of quarter-wave $\mathrm{TiO}_{2} /$ $\mathrm{SiO}_{2}$ reflective coatings were deposited on the front and the back facets, respectively. The LD device structure was lateral, with both the $\mathrm{p}$ - and the $\mathrm{n}$-contact pads on the same side of the device, as shown in Fig. 5a, b.

An optical power meter (Thorlabs PM121D) was used to measure the output power under $\mathrm{CW}$ electrical pumping. A fiber optic spectrometer (IdeaOptics FX4000) was utilized to measure the electrical luminescence spectra of the LDs. A PANalytical X'Pert Pro MRD highresolution X-ray diffractometer was employed for the double-crystal X-ray rocking curve measurements. A Hitachi HD-2700 field emission (FE)-STEM operated at $200 \mathrm{kV}$ and an FEI Quanta 400 FEG thermal field emission SEM operated at $10 \mathrm{kV}$ were used for taking the STEM and the SEM images, respectively.

\begin{abstract}
Acknowledgements
The authors are grateful for financial support from the National Key R\&D Program (Grant No. 2016YFB0400100, 2016YFB0400104), the National Natural Science Foundation of China (Grant Nos. 61534007, 61404156, 61522407, 61604168, and 61775230), the Key Frontier Scientific Research Program of the Chinese Academy of Sciences (Grant No. QYZDB-SSWJSC014), the Strategic Priority Research Program of the Chinese Academy of Sciences (Grant No. XDA09020401), the Science and Technology Service Network Initiative of the Chinese Academy of Sciences, the Key R\&D Program of Jiangsu Province (Grant No. BE2017079), and the Natural Science Foundation of Jiangsu Province (Grant No. BK20160401). This work was also supported by the open fund of the State Key Laboratory of Luminescence and Applications (Grant No. SKLA-2016-01), and the open fund of the State Key Laboratory on Integrated Optoelectronics (Grant No. IOSKL2016KF04, and IOSKL2016KF07). We are thankful for technical support from the Nano Fabrication Facility, Platform for Characterization \& Test, and Nano-X of SINANO, CAS.
\end{abstract}

\section{Author details}

'Key Laboratory of Nanodevices and Applications, Suzhou Institute of NanoTech and Nano-Bionics (SINANO), Chinese Academy of Sciences (CAS), Suzhou 215123, China. ${ }^{2}$ School of Nano Technology and Nano Bionics, University of Science and Technology of China, Hefei 230026, China. ${ }^{3}$ Accelink Technologies Co., Ltd, Wuhan 430205, China. ${ }^{4}$ State Key Laboratory of Luminescence and Applications, Changchun Institute of Optics Fine Mechanics and Physics (CIOMP), CAS, Changchun 130033, China

\section{Conflict of interest}

The authors declare that they have no conflict of interest.

Supplementary information accompanies this paper at https://doi.org/ 10.1038/s41377-018-0008-y.

Received: 22 August 2017 Revised: 10 January 2018 Accepted: 26 February 2018 Accepted article preview online: 1 March 2018

Published online: 13 June 2018

\section{References}

1. Denault, K. A., Cantore, M., Nakamura, S., DenBaars, S. P. \& Seshadri, R. Efficient and stable laser-driven white lighting. AIP Adv. 3, 072107 (2013).

2. Wierer, J. J. Jr, Tsao, J. Y. \& Sizov, D. S. Comparison between blue lasers and light-emitting diodes for future solid-state lighting. Laser Photonics Rev. 7, 963-993 (2013)

3. Nakamura, S. et al. High-power, long-lifetime InGaN/GaN/AlGaN-based laser diodes grown on pure GaN substrates. Jpn J. Appl. Phys. 37, L309-L312 (1998).

4. Wierer, J. J. Jr, Tsao, J. Y. \& Sizov, D. S. The potential of III-nitride laser diodes for solid-state lighting. Phys. Status Solidi C 11, 674-677 (2014).

5. Tsonev, D., Videv, S. \& Haas, H. Towards a $100 \mathrm{~Gb} / \mathrm{s}$ visible light wireless access network. Opt. Express 23, 1627-1637 (2015).

6. Oubei, H. M. et al. 4.8 Gbit/s 16-QAM-OFDM transmission based on compact 450-nm laser for underwater wireless optical communication. Opt. Express 23, 23302-23309 (2015)

7. Ichimura, I., Maeda, F., Osato, K., Yamamoto, K. \& Kasami, Y. Optical disk recording using a GaN blue-violet laser diode. Jpn J. Appl. Phys. 39, 937-942 (2000).

8. Motoki, K. et al. Growth and characterization of freestanding GaN substrates. J. Cryst. Growth 237-239, 912-921 (2002).

9. Dadgar A., et al. Metalorganic chemical vapor phase epitaxy of gallium-nitride on silicon. Phys. Status Solidi C 1583-1606 (2003).

10. Sun, Y. et al. Room-temperature continuous-wave electrically injected InGaNbased laser directly grown on Si. Nat. Photonics 10, 595-599 (2016).

11. Sun, Q. et al. GaN-on-Si blue/white LEDs: epitaxy, chip, and package. J. Semicond. 37, 044006 (2016)

12. Leung, B., Han, J. \& Sun, Q. Strain relaxation and dislocation reduction in AIGaN step-graded buffer for crack-free GaN on Si (111). Phys. Status Solidi C 11, 437-441 (2014).

13. Romanov, A. E. \& Speck, J. S. Stress relaxation in mismatched layers due to threading dislocation inclination. Appl. Phys. Lett. 83, 2569-2571 (2003).

14. Follstaedt, D. M., Lee, S. R., Allerman, A. A. \& Floro, J. A. Strain relaxation in AlGaN multilayer structures by inclined dislocations. J. Appl. Phys. 105, 083507 (2009).

15. Heying, B. et al. Role of threading dislocation structure on the x-raydiffraction peak widths in epitaxial GaN films. Appl. Phys. Lett. 68, 643-645 (1996).

16. Chierchia, R. et al. Microstructure of heteroepitaxial GaN revealed by x-ray diffraction. J. Appl. Phys. 93, 8918-8925 (2003).

17. Cherns, D., Henley, S. J. \& Ponce, F. A. Edge and screw dislocations as nonradiative centers in InGaN/GaN quantum well luminescence. Appl. Phys. Lett. 78, 2691-2693 (2001).

18. Nakamura, S. \& Fasol, G. The Blue Laser Diode: GaN Based Light Emitters and Lasers (Springer, Berlin Heidelberg, 1997).

19. Zhang, L. Q. et al. Confinement factor and absorption loss of AllnGaN based laser diodes emitting from ultraviolet to green. J. Appl. Phys. 105, 023104 (2009).

20. Nakamura, S. et al. Blue InGaN-based laser diodes with an emission wavelength of 450nm. Appl. Phys. Lett. 76, 22-24 (2000).

21. Li, Z. C. et al. Suppression of thermal degradation of InGaN/GaN quantum wells in green laser diode structures during the epitaxial growth. Appl. Phys. Lett. 103, 152109 (2013).

22. Van Daele, B., Van Tendeloo, G., Jacobs, K., Moerman, I. \& Leys, M. R. Formation of metallic In in InGaN/GaN multiquantum wells. Appl. Phys. Lett. $\mathbf{8 5}$ 4379-4381 (2004)

23. Queren, D. et al. Quality and thermal stability of thin InGaN films. J. Cryst. Growth 311, 2933-2936 (2009).

24. Duxbury, N. et al. Indium segregation in InGaN quantum-well structures. Appl. Phys. Lett. 76, 1600-1602 (2000).

25. Sugahara, T. et al. Role of dislocation in InGaN phase separation. Jpn. J. Appl. Phys. 37, L1195-L1198 (1998).

26. Massabuau, F. C. P. et al. Morphological, structural, and emission characterization of trench defects in InGaN/GaN quantum well structures. Appl. Phys. Lett. 101, 212107 (2012).

27. Massabuau, F. C. P. et al. Correlations between the morphology and emission properties of trench defects in InGaN/GaN quantum wells. J. Appl. Phys. 113, 073505 (2013).

28. Zhou K, et al. Thermal degradation of InGaN/GaN quantum wells in blue laser diode structure during the epitaxial growth. In Proceedings of the SPIE Volume 10244 (International Conference on Optoelectronics and Microelectronics Technology and Application, 102441×, Shanghai, China, 2017). 
29. Tian, A. Q. et al. Conductivity enhancement in AlGaN:Mg by suppressing the incorporation of carbon impurity. Appl. Phys. Express 8 , 051001 (2015).

30. Nakamura, S. The roles of structural imperfections in InGaN-Based blue lightemitting diodes and laser diodes. Science 281, 956-961 (1998).
31. Nakamura, S. et al. Room-temperature continuous-wave operation of InGaN multi-quantum-well structure laser diodes. Appl. Phys. Lett. 69, 4056-4058 (1996).

32. Nakamura, S. et al. High-power, long-lifetime InGaN multi-quantum-wellstructure laser diodes. Jpn J. Appl. Phys. 36, L1059-L1061 (1997). 